\title{
Planning Annuaulised hours when spike in demand exists
}

\author{
M R Sureshkumar* and V Madhusudanan Pillai
}

Department of Mechanical Engineering, National Institute of Technology Calicut, NIT Campus (P.O), Calicut 673 601, Kerala, India

\begin{tabular}{|c|c|}
\hline A R T I C L E I N F O & AB S TRACT \\
\hline $\begin{array}{l}\text { Article history: } \\
\text { Received } 25 \text { October } 2011 \\
\text { Accepted February, } 32012 \\
\text { Available online } \\
17 \text { February } 2012 \\
\text { Keywords: } \\
\text { Manpower planning } \\
\text { Annualised hours } \\
\text { Mixed integer linear programming } \\
\text { Holiday weeks } \\
\text { Flexible working }\end{array}$ & $\begin{array}{l}\text { Manpower planning using annualised hours is an effective tool where seasonal demand for staff } \\
\text { in industry exists. In annualised hours (AH) workers are contracted to work for a certain number } \\
\text { of hours per year. The workers are associated with relative efficiency for different types of tasks. } \\
\text { This paper proposes a Mixed Integer linear Programming (MILP) model to solve an annualised } \\
\text { working hours planning problem when spike in demand exists. The holiday weeks for the } \\
\text { workers are considered as partially individualised. If a worker has been assigned with more than } \\
\text { one type of working week in a week, this will be compensated with one or more holiday week. } \\
\text { The performance of the model is demonstrated with an example. It can be seen that this type of } \\
\text { modelling helps to meet the spikes in demand with less capacity shortage compared with one } \\
\text { working week in a week. }\end{array}$ \\
\hline
\end{tabular}

\section{Introduction}

In any industry, manpower planning has got utmost importance. It becomes more crucial when the seasonality in demand exists. Manpower planning under seasonal demand conditions may be difficult to tackle using normal manpower planning methods. For instance, when demand is more it leads to hectic overtime to workers where as on the other hand when demand is less it leads to underutilisation of workers. Annualised hours (AH) is a suitable method to tackle such situations. In this method, the employees will be contracted for total annual hours and these hours are distributed irregularly to meet the fluctuations in demand. The annual contracted hours will be based on the average weekly working hour. By this method employers will be able to meet the fluctuations in demand with a reduced overtime. The other advantage is that the training/subcontracting and inventory costs can be reduced. This method of manpower planning using AH has found successful in many organisation. For example Rhodia Consumer Specialities (Workforce logistics, 2007) introduced the annualised hours for staff scheduling and the benefits were that the consumer complaints fell by $25 \%$ and the service standard was improved, substantially. The results from the "Tesco" distribution (MacMeeking, 1995) showed that after the introduction of the AH system the stock levels had reduced to a large extent. AH may be implemented in organisation after proper consultation of the management with the worker's union. This will reduce the uncertainties regarding the $\mathrm{AH}$ scheme and will make the $\mathrm{AH}$ implementation * Corresponding author. Tel: +91 9483824097

E-mail: sureshmullanezhy@gmail.com (M. R. Sureshkumar)

(c) 2012 Growing Science Ltd. All rights reserved.

doi: 10.5267/j.ijiec.2012.02.004 
smoother. Any irregularities have to be negotiated properly followed by some sort of incentive and the incentive may be financial or non financial.

$\mathrm{AH}$ is always associated with holiday weeks. Holiday weeks are the weeks in which the workers will not work. There are different types of holiday weeks. First one is individualised holiday weeks and the second is partially individualised holiday weeks. In the individualised holiday weeks the holiday weeks are fixed after prior consultation with the workers. In partially individualised holiday weeks part of the holiday weeks are fixed after prior consultation with the workers and the remaining holiday weeks are considered as a decision variable. Under $\mathrm{AH}$ the weekly working hours can be taken from a finite set of weekly working hours associated with working week. For example, if in a planning horizon of 52 weeks the total working week is 46 and remaining 6 weeks are holiday weeks, then the weekly working hours and working weeks can be defined as follows. Weekly working hours of 25 hours of 15 weeks,35 hours of 21 weeks and 50 hours of 10 weeks. This implies the workers can work for 15 weeks with 25 hours and 35 hours with 21 weeks and 50 hours with 10 weeks so that the total working will be 46 .The type of working week in a week will be will be decided by the program. This type of finite set of weekly working hours is used in this paper.

There are a lot of papers are published in the area of annualised hours. Corominas et al. (2007a) explains an AH scenario in which the objective is to minimize the weighted sum of maximum relative capacity shortages and sum of weekly relative capacity shortages. The problem is solved using Mixed Integer Linear Programming method. Weekly working hours are taken from a finite set. In this paper, only one type of working week for a worker in one week exists. Workers are categorised depending on the skill which they are having and different relative efficiency is considered for performing different types of tasks. Annualisation of working hours for a firm working under shift scenario has also explored. Hung (1999a) tells how to schedule workers when the manpower requirement, number of operating days, and shift length change from period to period. Hung (1999b) suggested a method for scheduling the manpower for a multiple shift operation of a facility. A mixed integer linear programming model (Corominas et al., 2007b) is developed to solve the problem of planning the production and the working hours of a human team, which operates in a multi-product process considering all the relevant constraints. Planning $\mathrm{AH}$ with joint holidays suggested by Corominas et al (2004) shows a method for annualising using finite set of type of working hours. A simple three step approach was suggested by Azmat and Widmer (2004) for planning, scheduling and task assignment, which consider different situations for the problem being solved. In an annualised planning problem with finite set of weekly working hours and cross trained workers, the importance of decision support system is presented by Grabot and Letouzey (2000), which gives specification of a DSS for short-term manufacturing under annualised hours. The effect of relative capacity shortage when holiday weeks are partially individualised is explained by Kumar and Pillai (2009). Corominas et al. (2002) proposed an annualised hour problem using LP model where the objective is to minimise the cost of overtime, external workers and penalties associated with assigning workers to different type of jobs.

Planning annualised hours for workers can be done with one type of working hours in a week from a finite set of type of working week (Corominas et al., 2007a). When seasonal demand pattern exists this method give good solution. The present paper suggests a method to minimise the weighted sum of maximum relative capacity shortages and sum of weekly relative capacity shortages when sudden change or spikes in demand pattern exists. The novelty of this paper is that the MILP model suggested in this paper allows more than one type of working week in a week. When more than one type of working week in a week is performed by a worker he/she will be provided with additional holiday week. The holiday weeks are partially individualised.

The layout of this paper is as follows: Section 2 introduces the problem characteristics. Section 3 describes the model features. Section 4 presents an example problem and Section 5 provides the conclusion. 


\section{Problem Description}

Computing the solution for the planning problem, where abrupt change in demand exists in the demand pattern, involves the determination of the type of weekly working hours to be assigned from a finite set of weekly working hours (the working hours are taken from a finite set 25, 35, 50 hours) considered for each member of the worker. The demand is having a spike at week 27 as shown in Fig. 1.To meet this demand effectively in that particular week, the workers are permitted to take two types of working week. The allotment of extra working week for the workers is decided by the program. It is not necessary that all the workers will get two working weeks in that particular week. These two types of working week are permitted with an upper bound of working hours. In this problem the upper bound of working hours is fixed as 60 hours, which is the sum of least two types of weekly working hours in the finite set, i.e. $35+25=60$. Since they are doing extra working week in that particular week they are provided with compensation with extra holiday week. This extra holiday week will be decided by the program. The model has been solved using MILP programming.

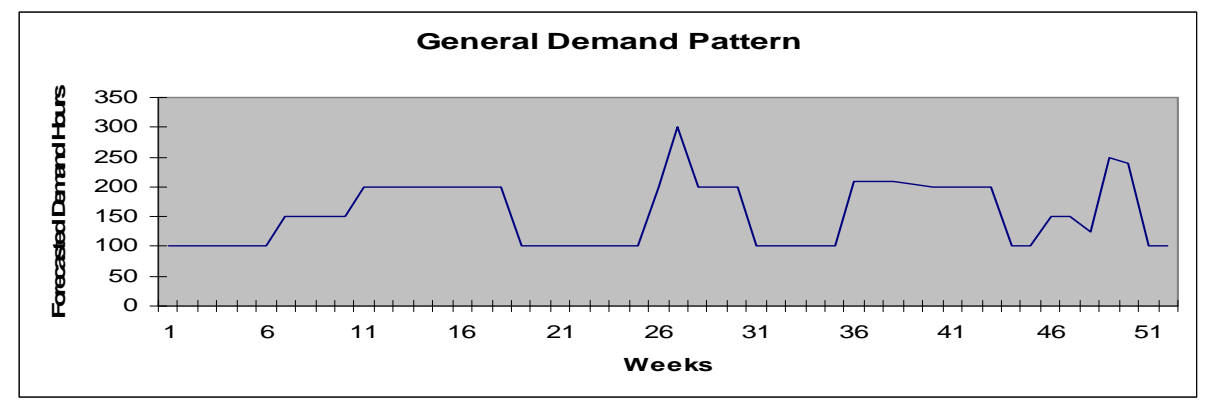

Fig. 1. General pattern demand

Since overtime and hiring of workers are not permitted the workers have to perform different types of tasks. This cross functionality is associated with relative efficiency for each type of task. The workers are classified into different categories depending on the skill they are having for different types of tasks. A relative efficiency 0.8 implies the workers will take 1/0.8 hours to complete the task. The characteristics of the problem are given below:

1) The numbers of working hours are taken from a finite set (e.g. 25, 35, 50 hours).

2) The average of working hours for a group of 12 consecutive weeks cannot be larger than 44 hours per week.

3) It is assumed that the average number of weekly working hours for a worker is 35.

4) The number of holiday weeks for each worker is constrained to six to determine the annual working hours. Hence, the number of annual working hours for a staff is $1610(46 \times 35)$.

5) There are different categories of workers and different types of tasks. Certain categories of workers can perform different types of tasks and thus have different relative efficiencies associated with them. This relative efficiency is given in Table 1.

Table 1

Relative efficiency

\begin{tabular}{lccc}
\hline & Task 1 & Task 2 & Task 3 \\
\hline Category 1 & 1 & 0.9 & 0.8 \\
Category 2 & 0 & 1 & 0.9 \\
Category 3 & 0 & 0.5 & 1 \\
\hline
\end{tabular}

6) Total capacity in hours is same as the demand requirement.

7) Overtime is not allowed.

8) Hiring temporary workers is not permitted. 
9) The numbers of workers are fixed according to the annual demand.

Since overtime and temporary workers are not allowed, the cost of the staff will be same for all feasible solutions. Thus to optimise the desired service level capacity has to be distributed. The effectiveness model is ascertained by solving the model with one working in a week. This is illustrated with an example problem in Section 4.

\subsection{Objective Function}

Objective function described here is same as that of Corominas et al. (2007a), which involves the minimisation of weighted sum of maximum capacity shortage and sum of weekly relative capacity shortages. The objective of any organisation will be the minimization of the overall cost, better customer satisfaction and maximisation of profit. As the service level improves, the customer satisfaction increases. The capacity of the organization is fixed as per the foreseen demand. If the required capacity is more than the actual capacity then the service level deteriorates and the customer will not be satisfied. If the relative capacity shortage, which is defined as the capacity shortage related to the required capacity is large then the demand cannot be met. On the other hand if the capacity shortage is a small part of the required capacity then the workers can meet the demand with a small extra effort. That is, the demand will be met with slightly reduced service quality.

The maximum relative capacity shortage, which has to be minimised, can be considered as objective function, thus optimising the service level during worst affected period. This function avoids large capacity shortages and tends to distribute capacity over the course of the year in a regular way. Even though, the function minimises the maximum capacity shortage, it is not giving consideration to periods where capacity shortages less than the maximum relative capacity shortage. For obtaining a small capacity shortage in every week, a secondary objective function, which is the sum of relative capacity shortages of various weeks, is considered. Now the objective function is defined as the weighted sum of these two functions. That is, the objective function minimises the weighted sum of two terms: (i) the maximum relative capacity shortages and (ii) the sum of weekly relative capacity shortages.

\section{The MILP Model}

The problem parameters and the notations used are as follows

$W$ - Set of staff members

$P$ - Number of weeks in the planning horizon

$G$ - Set of category of workers

$T$ - Set of types of tasks

$C_{i}$ - Category of worker $i$

$d 1$-the upper bound for weekly working hours for a worker in the week in which spike in demand exists

$p 1$-the week in which demand spike occurs.

$\tau_{g t}$ - Relative efficiency associated with category $g$ workers in the accomplishment of task of type $t$, $(\forall g \in G, \forall t \in T) ; \quad 0 \leq \tau_{g t} \leq 1$. Efficiency $\tau_{g t}=0$ indicates that category $g$ workers are not able to perform type $t$ task.

$H P$ - Set of types of working weeks expressed working hours.

$H P_{k^{-}}$The number of hours corresponding to working week type $k, k=1, \ldots|H P|$

$h_{i p}$ - Types of working weeks expressed in working hours, that worker $i$ can perform during week $p$, $(\forall i \in W, \forall p \in P)$

$n_{i k}$ - The total number of working hours over the year due to working week of type $k$ for the worker $i$, $(\forall i \in W, \forall k)$ 
$L, h_{L}$ - The average number of working hours in a group of $L$ consecutive weeks, which cannot be larger than $h_{L}$, (with $L=12$ and $h_{L}=44$ hours).

$f_{t p}$ - Working hours required for type $t$ task during week $p(\forall t \in T, p=1 \cdots P)$ considering the time that a worker (with relative efficiency of 1 ) would need to complete the task, and it is fixed according to forecasted demand and the expected service level.

$v_{i p}$ - Integer variable represents the number of types of working week to be assigned to worker $i$ during week $p$.

The decision variables are as follows:

$y_{i k p}$ - Binary variable that indicates whether worker $i$ performs a working week of type $k$ during week $p$ $(\forall i \in W, p=1 \cdots P)$

$a_{g t p}$ - Non-negative real variable that indicates the number of working hours that category $g$ employees dedicate to task $t$ during week $p$ ( $\forall g \in G, \forall t \in T, \forall p \in P \mid \tau_{g t}>0$ )

$s_{t p}$ - Non-negative real variable that indicates the capacity shortage for task $t$ during week $p$ $(\forall t \in T, p=1 \cdots P)$;

$S$ - Non-negative real variable that indicates the maximum shortage, as related to the demand

$$
\alpha=0.99, \beta=\frac{0.01}{P \cdot|T|}
$$

\section{Model}

$\min Z=\alpha S+\beta \sum_{\forall t \in t} \sum_{p=1}^{P} \frac{s_{t p}}{f_{t p}}$

subject to

$$
\begin{aligned}
& S \geq \frac{s_{t p}}{f_{t p}} \quad \forall t \in T, p=1 \cdots P \\
& \sum_{\forall p} y_{i k p}=n_{i k}, \quad \forall i \in W, \forall k \\
& \sum_{\forall k} y_{i k p} \leq v_{i p} \quad \forall i \in W ; \quad p=1 \cdots P \\
& \sum_{\forall k} y_{i k p} H P_{k} \leq d 1, \quad \forall i \in W, p=p_{1} \\
& \sum_{(\forall g \in G)\left(\tau_{g t}>0\right)} \tau_{g t} a_{g t p}+s_{t p} \geq f_{t p} \quad \forall t \in T, p=1 \ldots P \\
& \underset{\forall t \in T \mid\left(\tau_{g_{t}}>0\right)}{a_{g t p}}=\sum_{\left(\forall i \in W \mid C_{i}=g\right)} \sum_{\forall k} H P_{k} y_{i k p}, \\
& \forall g \in G, \quad p=1 \ldots P \\
& \sum_{p=j-L+1}^{j} \sum_{\forall k} H P_{k} y_{i k p} \leq h_{L} \times L, \forall i \in W, j=L \ldots P \\
& y_{i k p}=\{0,1\} \text {; } \\
& a_{g t p}, s_{t p}, S \geq 0
\end{aligned}
$$

The objective function (1) minimizes the weighted sum of: (i) the maximum relative capacity shortage and (ii) the sum of weekly relative capacity shortages, with $\alpha, \beta>0$;

The model is formulated based on the following constraints: 
(2) ensures effect of maximum relative capacity shortage; (3) assigns the required number of type of working weeks for each worker as stipulated in the contract; (4) assigns the required number of type of working week as per the requirement;(5) The total working hour in a week where the spike in demand occurs cannot be more than d1 hours (6)demand requirements; (7) equalise the time allotted for all types of task in a week with the time assigned for all category of worker in the same week; (8) ensures the contractual condition that the average time assigned over a consecutive $L$ weeks is less than $h_{L}$.(9) and (10) are binary and nonnegative constraints respectively.

\section{Example Problem}

The model is demonstrated with an example. The basic data used for the problem are as follows:

- $|W|=$ staff sizes of 5 workers.

- $P=52$ (considering max 46 working weeks and 6 holiday weeks). The model assigns the holiday weeks for each worker and seven is the minimum number of holiday weeks.

- When spikes in the demand pattern exists, a worker will be asked to take an additional type of working hours, from the previously defined set of type of weekly working hours in a week for which the worker will be compensated with an additional holiday week.

- $\quad$ There are three categories of workers.

- There are three types of working weeks for each worker. The number of hours of each type and the number of weeks of each type in an year are:

- Type 1 the number of hours $H P_{1}=25$ hours and $n_{i 1}=15$ weeks

- Type 2 the number of hours $H P_{2}=35$ hours and $n_{i 2}=21$ weeks

- Type 3 the number of hours $H P_{2}=50$ hours and $n_{i 2}=10$ weeks

- There are three types of tasks.

- The relative efficiency for the worker category for different tasks is shown in the Table 1.

- $\quad d 1=60$ hours and $p 1=$ week 27 .

The demand pattern considered for the problem is shown in Fig. 1. For instance the demand has a spike in the week 27 and the magnitude of this demand is 300 hours. When the annualisation is done using more than one working week in that particular week, the assigned hours after considering relative efficiency is increased to 274 hours and due to that the shortage hours is reduced to $300-274=26$ hours in that particular week. Fig. 2 shows the demand pattern, assigned hours considering the relative efficiency and capacity shortage when two working weeks in a week are considered.

Table 2

Holiday weeks

\begin{tabular}{cccc}
\hline Worker & Individualised Holiday weeks & Variable Holiday Weeks & Total number of holiday weeks \\
\hline 1 & 1,2 & $31,35,49,51$ & 6 \\
\hline 2 & 3,4 & $20,24,44,45,48$ & 7 \\
\hline 3 & 5,6 & $19,21,32,34,52$ & 7 \\
\hline 4 & 7,8 & $23,24,33,34,50$ & 7 \\
\hline 5 & 9,10 & $2,22,24,31,51$ & 7 \\
\hline
\end{tabular}

Table 2 shows the details of holiday weeks. From the table it can be seen that for workers 2, 3, 4 and 5 the total holiday weeks are seven. This implies that these workers have taken extra working week in week number 27 so that they have been awarded extra holiday weeks. Fig. 3 shows the demand, assigned hours and capacity shortage when one working week is considered in a week. If the annualisation is done using one working week in that particular week, which will result in a shortage of 300-241=59 hours. The shortage values for different manpower under the same demand pattern are given in Table 3. In all the cases it is seen that the shortage values are less when more than one working 
week is considered. So when sudden demand occurs during a particular period in planning horizon this type of modelling will produce better result.

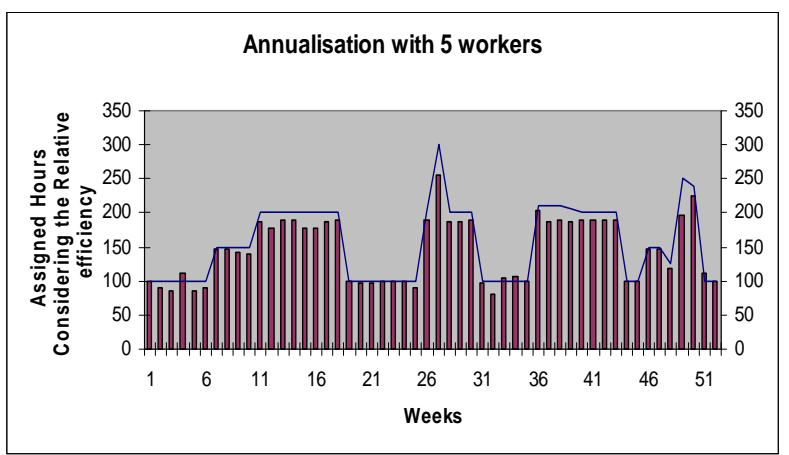

Fig. 2. Required capacity, assigned capacity and shortage profiles for the 5 workers considering more than one type of working week in week 27

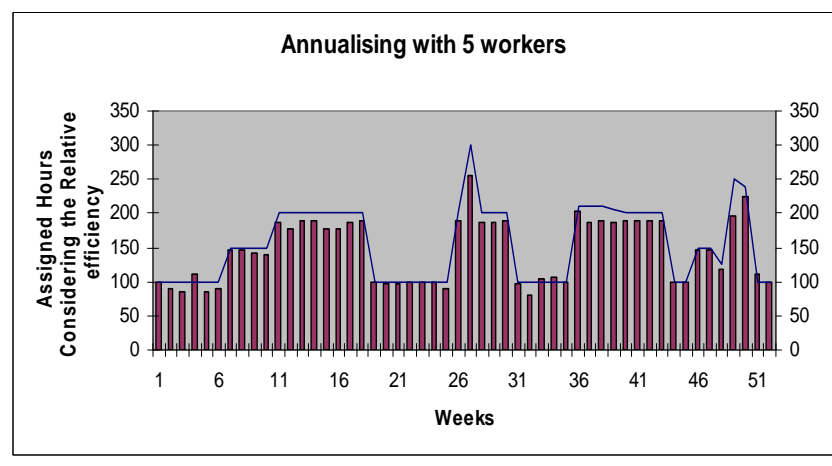

Fig. 3. Required capacity, assigned capacity and shortage profiles for the 5 worker problem considering one type of working week in week 27

\section{Table 4}

Demand and shortage hour details in week 27

\begin{tabular}{lllllll}
\hline \multirow{2}{*}{ Worker } & \multicolumn{4}{l}{ More than one working week in a week } & \multicolumn{3}{l}{ One working week in a week } \\
\cline { 2 - 7 } & Demand (hours) & Assigned hours & Shortage(Hours) & Demand Hours & Assigned Hours & Shortage(Hours) \\
\hline 5 & 300 & 274 & 26 & 300 & 241 & 59 \\
10 & 600 & 547 & 53 & 600 & 483 & 117 \\
50 & 3000 & 2796 & 204 & 3000 & 2418 & 582 \\
250 & 15000 & 13964 & 1036 & 15000 & 12094 & 2906 \\
\hline
\end{tabular}

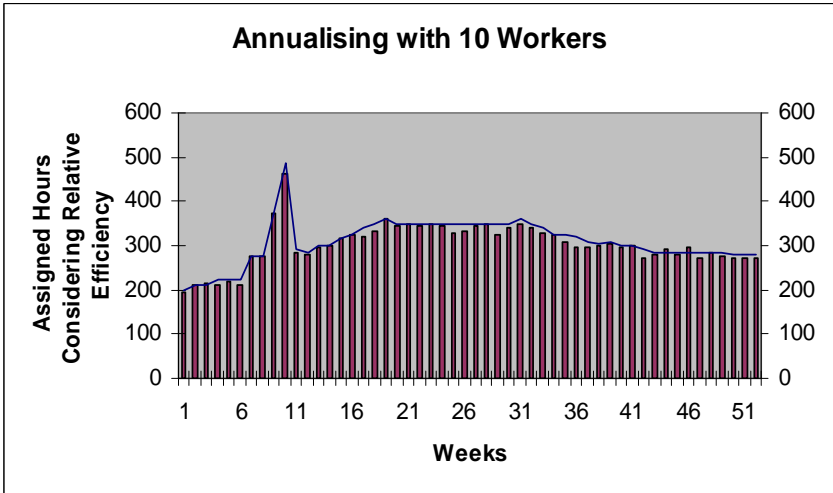

Fig. 4. Required capacity, assigned capacity and shortage profiles for the 10workers considering more than one type of working week in week 10

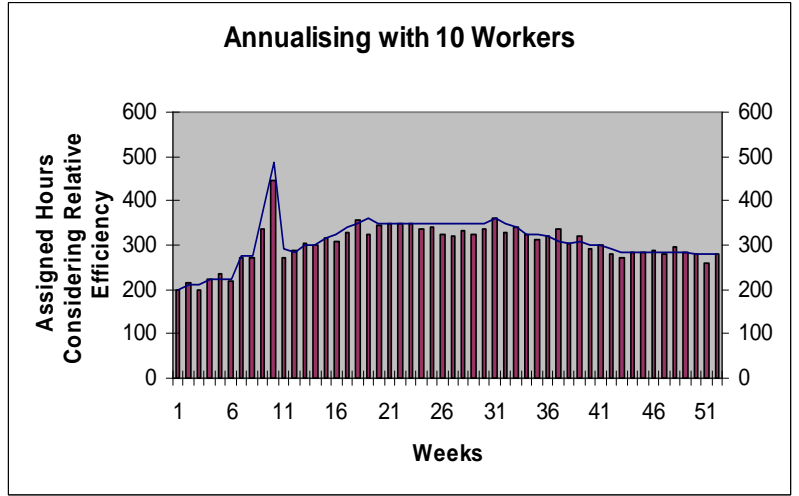

Fig. 5. Required capacity, assigned capacity and shortage profiles for the 10 workers problem considering one type of working week in week 10

Fig. 4 shows the demand requirement, for a different demand pattern with a single spike when more than one working week is considered. The procedure of modelling is same as described in this section but the relative efficiency and the week in which the demand spike occurs is different. In this case the demand spike occurs in week 10 and the relative efficiency considered for the workers is given in Table 3.The demand requirement in week 10 is 487 hours and the assigned hours after considering relative efficiency is 460 hours. So the shortage is $487-460=27$ hours. But when only one working week is considered the shortage is $487-447=40$ hours. Fig. 5 shows the demand requirement, for a different demand pattern with a single spike when one working week is considered. Table 4 shows the capacity shortage for different manpower using this demand type. 
Table 3

Relative Efficiency

\begin{tabular}{llll}
\hline & Task 1 & Task 2 & Task 3 \\
\hline Category 1 & 1 & 0.9 & 0 \\
Category 2 & 0 & 1 & 0.9 \\
Category 3 & 0 & 0 & 1 \\
\hline
\end{tabular}

Table 4

Demand and shortage hour details (in week 10 with new demand pattern)

\begin{tabular}{lllllll}
\hline \multirow{2}{*}{ Worker } & \multicolumn{5}{l}{ More than one working week in a week } & \multicolumn{4}{l}{ One working week in a week } \\
\cline { 2 - 7 } & Demand hours & Assigned hours & Shortage (Hours) & Demand Hours & Assigned Hours & Shortage(Hours) \\
\hline 10 & 487 & 460 & 27 & 487 & 447 & 40 \\
50 & 2435 & 2366 & 69 & 2435 & 2133 & 302 \\
\hline
\end{tabular}

\section{Conclusions}

Generally, AH scheme allows an organisation to schedule manpower according to the fluctuation in demand. Usually, the AH scheme is implemented with a finite set of type of working weeks and the finite set approach has also been incorporated. To accommodated spikes in the demand pattern the finite set approach is modified. The capacity shortage in the particular week where spike in demand occurs is less when modified approach is used which shows the suitability of modified approach. The effect of number of working weeks on capacity shortages will be another area to be explored under spike in demand situation.

\section{References}

Workforce logistics, Annual hours case study-Rhodia. Available online at: www.workforcelogistics.com/Case_study_Annual_Hours_at_Rhodia.htm. (accessed on 11-09-2007).

MacMeeking, J. ( 1995). Why Tesco’s new composite distribution needed annual hours. International Journal Retail Distribution Management, 23, 36-38.

Corominas, A., Lusa, A., \& Pastor, R. (2007a). Planning annualised hours with a finite set of weekly working hours and cross trained workers. European Journal of Operational Research, 176, 230239.

Hung, R. (1999a). Scheduling a workforce under annualised hours. International Journal of Production Research, 37(11), 2419-2427.

Hung, R. (1999b). A multiple-shift workforce scheduling model under annualised hours. Naval Research Logistic, 46, 726-736.

Corominas, A., Lusa, A., \& Pastor, R. (2007b). Using a MILP model to establish a framework for an annualised hours agreement. European Journal of Operational Research, 177, 1495-1506.

Corominas, A., Lusa, A., \& Pastor, R. (2004). Planning annualised hours with a finite set of weekly working hours and joint holidays. Annals of Operations Research, 128, 217-233.

Azmat, C.S., \& Widmer, M. (2004).A case study of single shift planning and scheduling under annualised hours: A simple three step approach. European Journal of Operational Research. 153 (1), 148-175.

Grabot, B., \& Letouzey, A. (2000). Short-term manpower management in manufacturing systems: New requirements and DSS prototyping. Computers in Industries, 3(1), 11-29.

Suresh Kumar, M.R, \& Pillai V. M, (2009). Planning Annualised Hours with Partially Individualised Holiday Weeks. International Journal of Operations and Quantitative Management, 15, 173-188.

Corominas, A., Lusa, A., \& Pastor, R. (2002) Using MILP to plan annualised working hours. Journal of Operational Research Society, 53, 1101-1108. 